\title{
Measuring Contributions to Mass Resolving Power in Atom Probe Tomography
}

\author{
E. Oltman, T.F. Kelly, T.J. Prosa, D. Lawrence, and D.J. Larson \\ Cameca Instruments, Inc., 5500 Nobel Drive, Madison, WI 53711
}

The mass resolving power (MRP) of a time-of-flight atom probe is a critical parameter that impacts such basic operation as elemental discrimination and analytical sensitivity. Yet, to date, it is illdefined and is subject to misinterpretation. There is a need for standard measurement and reporting of MRP values in atom probe tomography such that values from different instruments and different materials can be quantitatively and directly compared. This paper proposes a method and set of standard definitions to resolve this difficulty. Furthermore, the methods described here can reveal sources of instrumental problems like voltage fluctuations that can be a diagnostic tool.

A first-order analysis of the kinematics of a straight-flight-path atom probe gives $t^{2}=(\mathrm{m} / \mathrm{n}) *\left(\mathrm{l}^{2} / 2 \mathrm{eV}\right)$ where $t$ is time of flight, $m$ is ion mass, $n$ is charge state, $l$ is flight path length, $e$ is the elementary charge unit, and $V$ is the total flight acceleration potential [1]. We will restrict this discussion to instruments that do not have a reflectron. Error propagation analysis for flight time gives the components of the time spread, $\Delta t$ :

$$
\left(\frac{\Delta t}{t}\right)^{2}=\frac{1}{4}\left(\frac{\delta V}{V}\right)^{2}+\left(\frac{\delta l}{l}\right)^{2}+\left(\frac{\delta t}{t}\right)^{2}
$$

where $\delta V^{2}=\delta V_{i}^{2}+\delta V_{e}^{2}$ is the voltage term which encompasses both instrumental issues associated with unmeasured voltage ripple, $\delta V_{i}$, and the energy spread, e $\delta V_{e}$, of the evaporation process expressed as a voltage, $\delta V_{e} ; \delta l^{2}=\delta l_{g}{ }^{2}+\delta l_{p}{ }^{2}$ is the flight-path-length uncertainty with a global geometric term, $\delta l_{g}$, and a local pore term, $\delta l_{p}$, which is due to ions entering microchannel plate pores at different locations in the pore; and $\delta t^{2}=\delta t_{t}^{2}+\delta t_{d}{ }^{2}$ is the timing uncertainty which is a quadratic sum of the timer-hardware uncertainty, $\delta t_{t}$, which can be estimated, and $\delta t_{d}$, which is the ion time-ofdeparture spread. Since $\mathrm{MRP}=m / \Delta m=t / 2 \Delta t$, it is apparent that when $\delta V$ and $\delta l$ are small then $\Delta t$ should not depend on flight time and MRP should increase with $t$ which varies with $\sqrt{m / n}$ and $\sqrt{1 / V}$ for a given $l$. Thus if mass resolving power is to be compared from one experiment to the next, it must be reported at standard values of these parameters, at least. For example, when ion energy variations are negligible as should occur in laser pulsing, measurement of MRP at $m / n=61.3$ $\left({ }^{184} \mathrm{~W}^{+++}\right)$should give values that are $\sqrt{61.3 / 27}=1.51$ times greater than the value measured at $m / n=27\left({ }^{27} \mathrm{Al}^{+}\right)$. However, this is not always observed in practice. It is important then to assess the cause which is likely a non-negligible $\delta V$ term.

Fig. 1 is a map of $\Delta \mathrm{t}(\mathrm{FWHM})$ across the detector for ${ }^{27} \mathrm{Al}^{+}$data on a straight-flight-path Cameca LEAP4000X Si instrument. The geometric contributions to $\delta l$ can be modeled and the lateral spatial resolution of the detector, $\delta r$, is determined as a model parameter. Near the center is an area where $\delta l_{g}$ contributions become negligible. This minimum- $\delta l$ area is displaced from the center due to the bias angle of the microchannel plate pores: an effect which is contained in the model and can be removed from the data. Similarly, we can take $\delta t_{t}$ to be known, small, and constant ( $\left.\delta t_{t} \approx 0.075 \mathrm{~ns}\right)$.

Fig. 2 shows measured MRP as a function of flight time: eight measurements are made at each of 90 $\mathrm{mm}$ and $160 \mathrm{~mm}$ flight path length. Each measurement consists of 4 values: average flight time, average voltage, central flight path length and timing uncertainty. A global least squares fit to these 16 quartets of data was performed for the minimum- $\delta l$ area in Fig. 1 in each case with fit parameters $\delta V, \delta l$, and $\delta t$ in Eq. 1. Specimen-geometry variations may account for the scatter in the data. 
In Fig. 2, MRP decreases with increasing $t$ which must be due to a significant energy spread. The model finds a best fit to the parameters as listed in Table 1. The $\delta V$ term makes a greater contribution (lowers MRP) at larger $t$. It is important to distinguish whether the voltage spread, $\delta V$, or the normalized voltage spread, $\delta V / V$, is constant. The difference is dramatically evident in Fig. 3. Measurements at low voltage (long flight time) are most sensitive to this distinction and can reveal important instrumental limitations. The model parameters that result in the best fit are consistent with a constant $\delta V$. With these results the model in Fig. 3 matches well the data.

Reference

[1] M.K. Miller et al., Atom Probe Field Ion Microscopy (1996) Oxford University Press.

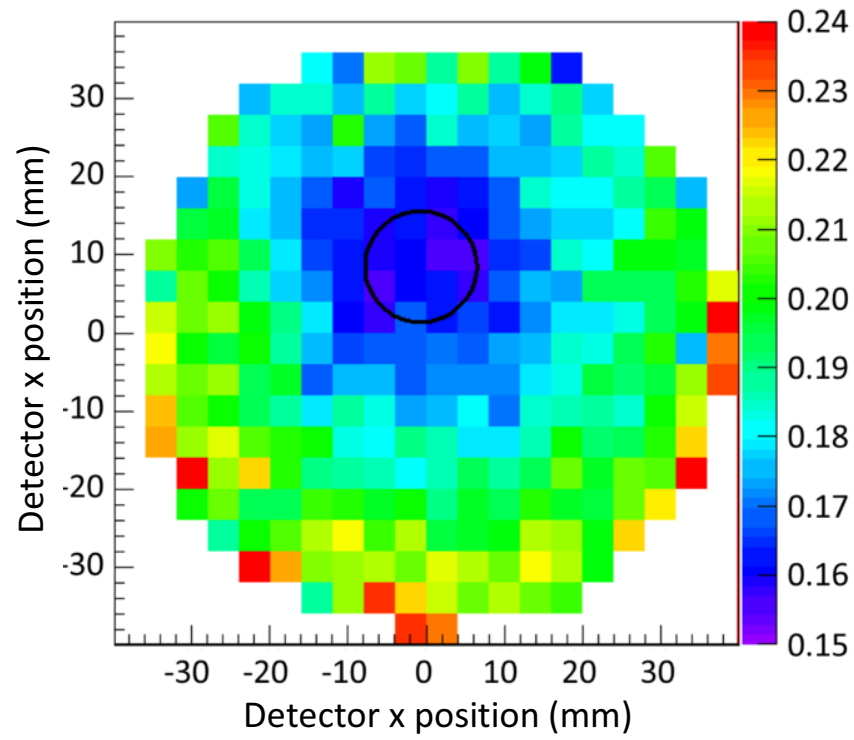

FIG. 1 Timing uncertainty, $\Delta \mathrm{t}$ (FWHM), measured with position on the detector. Righthand scale is timing uncertainty in ns.

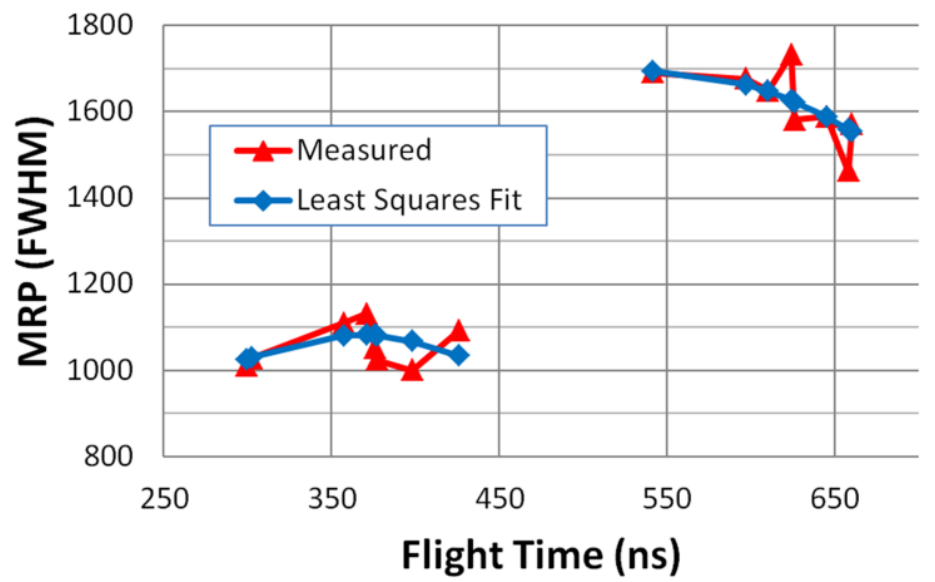

FIG. 2 Measured data with a least-squares fit of the several variables shown in Fig. 3. The groupings are due to two flight path lengths. The individual data points are due to different specimens running at different voltages.

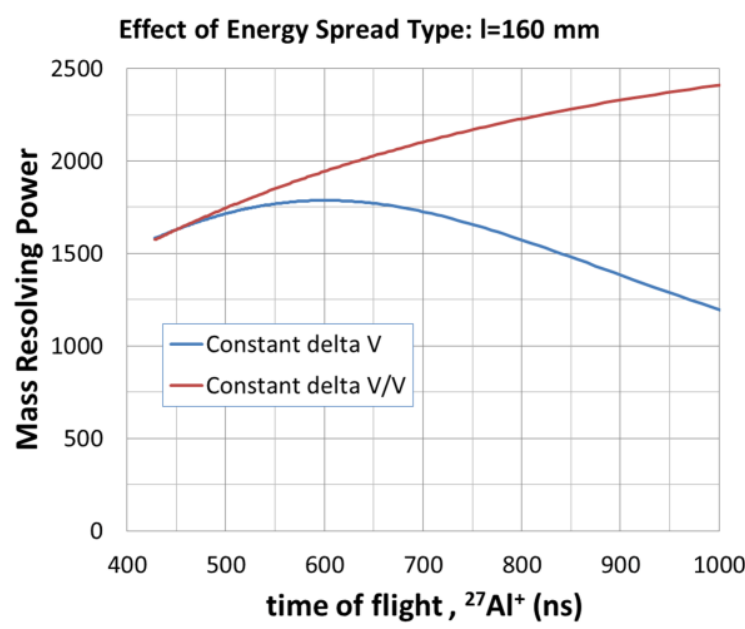

FIG. 3 Effect of energy-spread type on expected MRP. When $\delta \mathrm{V}$ is constant, the MRP decreases markedly at lower operating voltages (greater $\mathrm{t}$ ).

\begin{tabular}{|l|l|}
\hline Parameter & Value \\
\hline$\delta V$ & 2.72 volt \\
\hline$\delta l$ & $0.025 \mathrm{~mm}$ \\
\hline$\delta t$ & $0.114 \mathrm{~ns}$ \\
\hline
\end{tabular}

Table 1 Values of the adjustable parameters in the least squares fit of measured data with Eq. 1. 